\title{
Y(4260) and possible charmonium assignment
}

\author{
Felipe J. Llanes-Estrada* \\ Departamento Física Teórica I, Universidad Complutense de Madrid, 28040 Madrid, Spain
}

(Received 6 July 2005; published 30 August 2005)

\begin{abstract}
The newly reported Y(4260) becomes the second most massive state in the charmonium family. We argue that it displaces the $\psi(4415)$ as the (largely) $4 s$ vector charmonium state, recall $s-d$ wave interference to explain the lack of a signal in $e^{-} e^{+} \rightarrow$ hadrons and suggest some further study avenues that can exclude exotic meson assignments. The absence of a $J / \psi K K$ mode can be understood, beyond phase space suppression, to be a consequence of chiral symmetry. We also provide a model calculation in this sector showing that, although forcing the fit somewhat (which suggests a small sea quark wave function component), the state can be incorporated in a standard scheme.
\end{abstract}

DOI: 10.1103/PhysRevD.72.031503

PACS numbers: 12.39.Pn

\section{REPORTED PROPERTIES OF Y(4260) AND INTERPRETATION}

Yet another unexpected state has arisen from spectroscopy at the $B$ factories. The $\mathrm{Y}(4260)$ detected at $B A B A R$ [1] adds to the tower of $\psi$ states, but unexpectedly, below the last known resonance in this tower. It is common quark model wisdom that vector quarkonium states come in pairs, due to the approximately equal energy cost of a $d$ wave and a radial excitation. A first glance to the new spectrum already suggests that this state should be assigned the nomenclature $\psi\left(4^{3} S_{1}\right)$. Of course, $L$ is not a good quantum number and a $d$ wave will be present to some extent. More important is the increased splitting and lower mass ( $200 \mathrm{MeV}$ below the prediction of Godfrey and Isgur [2]) that calls for a sizable sea quark wave function component. But this mass itself can be made consistent with a quark model by softening the string tension at large distances. And again the mass is not far from the prediction in [3] (4300).

The width of the state, reported to be about 90 (20) MeV is also consistent with expectations from the quark model. For example, Barnes, Godfrey, and Swanson [4], under the assumption that the $\psi(4415)$ was the $4^{3} S_{1}$, evaluated its width at $77 \mathrm{MeV}$. A value somewhat smaller is to be expected at the lower mass 4260, but consistent with data. Moreover we agree with the quantum numbers proposed by the experimental collaboration based on its initial state radiation production, also consistent with the emission of an even number of pions and chiral symmetry expectations.

The discovery final state $J / \psi \pi \pi$ is also typical of $n^{3} S_{1} \rightarrow 1^{3} S_{1}$ transitions that have been studied in detail for the $\psi^{\prime}$ and $\psi(3770)$ [5]. Their branching fractions differ from roughly $50 \%$ to $0.5 \%$ by 2 orders of magnitude. This can be understood as $d$-wave suppression in the $\psi(3770)$

\footnotetext{
*On assignment at: Theory Group, Stanford Linear Accelerator Center, 2571 Sand Hill Road, Menlo Park, CA 94025, USA.

Electronic address: fllanes@fis.ucm.es
}

and does not apply to the present case of the Y(4260). We should be comparing instead with the branching fraction of the $3 S$ state, unfortunately unknown to us. If we use the $Y$ system as a guidance,

$$
\frac{\Upsilon^{\prime \prime \prime} \rightarrow \Upsilon \pi \pi}{\Upsilon^{\prime} \rightarrow \Upsilon \pi \pi} \simeq 0.1-0.2
$$

indicates that we should expect a branching fraction $B(\psi(4 S) \rightarrow J / \psi \pi \pi) \simeq 2 \%-4 \%$ (added phase space cannot compensate the much smaller wave function overlap). From the reported

$$
B(Y \rightarrow J \psi \pi \pi) \Gamma\left(Y \rightarrow e^{-} e^{+}\right) \simeq 4-7 \mathrm{eV},
$$

we can thus estimate $\Gamma\left(Y \rightarrow e^{-} e^{+}\right) \simeq 0.2-0.35 \mathrm{keV}$. On the basis of the model calculation below we would expect a somewhat larger lepton width.

The absolute width to $J / \psi \pi \pi$ is conceivably $1-2 \mathrm{MeV}$, a factor of 4 larger than the $2 S$ state. This is a novel effect that could be explained within a $c \bar{c}$ model by invoking a small admixture of a four quark state, or in another language, a nonvanishing coupling to the $J / \psi f_{J}$ channel that is open for this decay. This is an interesting calculation for future work.

The lack of a signal in the $J / \psi K K$ channel is a consequence of phase space suppression that might be very enhanced due to chiral symmetry. If the decay $Y \rightarrow$ $J / \psi M_{1} M_{2}$ was due exclusively to an $\mathrm{SU}(3)$ symmetric constant vertex, then the ratio between signals in the $J / \psi K K$ mode and the $J / \psi \pi \pi$ mode would be

$$
\frac{\int_{m_{K}}^{M_{Y}-m_{K}-m_{J / \psi}} d E_{1} \int_{m_{K}}^{M_{Y}-E_{1}} d E_{2}}{\int m_{\pi}^{M_{Y}-m_{\pi}-m_{J / \psi}} d E_{1} \int_{m_{\pi}}^{M_{Y}-E_{1}} d E_{2}} \simeq \frac{1}{4}
$$

that could very well be observed with increased statistics. On the other hand, in the case of a very convergent chiral expansion, where the first order coupling for the outgoing meson pair would dominate the matrix element, we would have an extra suppression factor 


$$
\frac{f_{\pi}^{4}\left(p_{K_{1}} \cdot p_{K_{2}}\right)^{2}}{f_{K}^{4}\left(p_{\pi_{1}} \cdot p_{\pi_{2}}\right)^{2}} \simeq \frac{f_{\pi}^{4} m_{K}^{4}}{f_{K}^{4} E_{\pi_{1}}^{2} E_{\pi_{2}}^{2}}
$$

lowering the ratio, after phase space integration, to about $0.3 \%$. This number is controlled by the high momentum phase space in the two pion decay channel and is very sensitive to higher order terms in a chiral expansion. We urge experimental collaborations to quote bounds on the $J / \psi K K$ channel, as it teaches us about the quality of chiral theory applied in an otherwise very nonperturbative domain [the doublet $\psi(4 S)$ and $\psi(3 D)$ are the highest known excitations of the QCD string].

The $V K K$ signal in a vector to vector transition would be interesting by itself because in the light sector this channel is closed for the $\rho(1450), \rho(1700), \omega(1650), \phi(1680)$, and also low-lying $\psi$ states.

The isospin of Y(4260) has not been reported, as no signal is yet observed in the more difficult $J / \psi \pi_{0} \pi_{0}$ channel (where $I=0$ requires a signal half of what was found in the charged mode). A signal in the $J / \psi \pi^{ \pm} \pi^{0}$ channel in $B$ decays would also establish nonzero isospin and rule out the charmonium assignment.

\section{ACCOMMODATING THE Y(4260) IN THE SPECTRUM}

To see how this new resonance fits in a standard Hamiltonian diagonalization, we recompute the vector charmonium spectrum in the $H_{\text {eff }}$ modeling QCD in Coulomb gauge described at length in [6-8].

The random phase approximation (RPA) diagonalization involves four parameters, largely constrained from other Fock space sectors. We employ a current $m_{c}$ that is dressed by means of the BCS gap equation for $H_{\text {eff }}$. The Coulomb gauge kernel is replaced by a momentum-space Cornell-type potential

$$
V(q)=-\frac{8 \pi \sigma}{q^{4}}-\frac{\alpha_{s}}{q^{2}}
$$

with the Coulomb tail cut off exponentially at a momentum of $5 \mathrm{GeV}$. The resulting spectrum for $m_{c}(2 \mathrm{GeV})=$ $1 \mathrm{GeV}, \alpha_{s}=0.6, \sqrt{\sigma}=350 \mathrm{MeV}$, that are typical for this model (with the string tension somewhat lower) is given in Table I. The new state is somewhat too light with respect to the pure $q \bar{q}$ assignment, which seems to

TABLE I. Coulomb gauge $H_{\text {eff }}$ RPA and updated experimental spectrum [9] incorporating the new state.

\begin{tabular}{lccc}
\hline \hline State & Calc. $M(\mathrm{MeV})$ & State & Calc. $M(\mathrm{MeV})$ \\
\hline$J / \psi(3097)$ & 3093 & $\psi(4160 \pm 20)$ & 4134 \\
$\psi(3686)$ & 3662 & $\mathrm{Y}(4260)$ & 4347 \\
$\psi(3770)$ & 3778 & $\psi(4415 \pm 10)$ & 4421 \\
$\psi(4040)$ & 4042 & & 4597 \\
& & & 4661 \\
\hline \hline
\end{tabular}

be the trend for the other novel states in charmonium spectroscopy above the charmed threshold. This is even after model readjustment (lowering the string tension and increasing the contribution of the Coulomb potential to avoid generating too light masses for the low-lying states). The calculation includes $s-d$ wave mixing and forwardbackward RPA propagating wave functions. We employ only the dominant $\gamma_{0} \gamma_{0}$ Hamiltonian, without including effects from the transverse gluon exchange $\alpha \cdot \alpha$, but the comparison with the charmonium states is fair. We obtain the $s$-wave lepton widths [10] $\Gamma_{e^{-}}^{n S} e^{+}(\mathrm{keV}) 4.4,2.3,1.9$, 0.98 , that show that the wave function is being artificially forced to shorter distances to accommodate the new state, and the widths of the higher radial excitations are larger than data by about a factor 2 .

In Fig. 1 we report a calculation of $D^{ \pm}, D_{s}^{ \pm}$production in $e^{-} e^{+}$scattering. We include a QCD background to account for the light quark production, and $D, D_{s}$ meson form factors that include the effect of the various $\psi$ resonances. We also recall [2] that the couplings of the $s$ - and $d$-wave $q \bar{q} 1^{--}$mesons decaying to a pseudoscalar pair have different signs. This interference could explain why

\section{$\mathrm{D}$, Ds production in the high charmonium region}

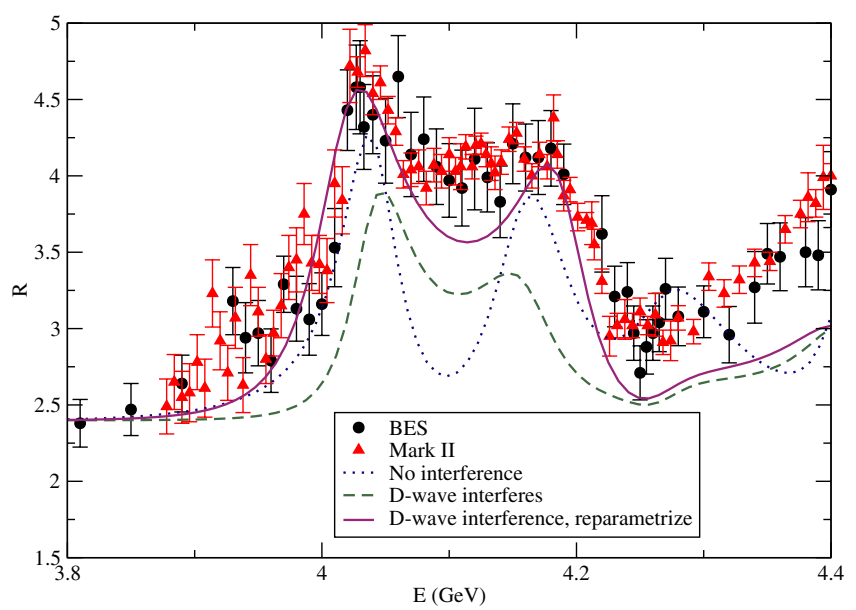

FIG. 1 (color online). Effect of a new charmonium state on $D^{+} D^{-}, D_{s}^{+} D_{s}^{-}$production at $4260 \mathrm{MeV}$, with parameters from the $B A B A R$ observation. We employ standard Particle Data Group parameters for the known resonances (dotted line). For comparison, the total $R\left(e^{-} e^{+} \rightarrow\right.$ hadrons) is given. The nonresonant $D, D_{s}$ production as well as light quark production (at the 2.4 level) is also added to the calculation for a more meaningful comparison. Observe that the spectrum looks qualitatively much better if the $d$-wave resonances $\psi(4160)$ and $\psi(4440)$ are taken to have negative coupling to the double pseudoscalar channel, as calculated by Godfrey and Isgur (dashed line). Finally, reparametrizing the resonances with $M_{\psi(3 s)}=$ $4.02 \mathrm{GeV}, \quad \Gamma_{\psi(3 s)}=80 \mathrm{MeV}, \quad \Gamma_{\psi(3 s)}^{e^{-} e^{+}}=1.5 \mathrm{keV}, \quad M_{\psi(2 d)}=$ $4.19 \mathrm{GeV}$, and $\Gamma_{\psi(2 d)}^{e^{-} e^{+}}=1.4 \mathrm{keV}$ arbitrarily fits total $D$ production, although a large fraction should proceed by an intermediate $D^{*}$. 
the $\mathrm{Y}(4260)$ has not been seen in past searches, as the $s-d$ interference washes it out of the $D D, D_{s} D_{s}$ spectrum and it must decay partly into other channels. Given that a large fraction of the produced $D$ mesons are decaying $D^{*}$ [11], this process needs further study. The fact that large interferences appear in the charmonium spectrum was already pointed out in [12].

\section{HOW TO EXPERIMENTALLY RULE OUT EXOTIC ASSIGNMENTS}

We should also consider the possibility of assigning the newly found Y(4260) to an exotic multiplet. The new charmonium and charmed spectroscopy has yielded several candidates for cryptoexotic tetraquarks, hidden exotics that have conventional quantum numbers but whose properties might reveal a large sea quark component. Recent arguments to classify the various candidates can be found in the literature, for the $X(3872)$ [13], $D_{S J}(2632)$ [14], $D_{S}(2320)$ [15,16], or $D_{S}(2308),(2317)$ [17-19]. An unlikely possibility for $\mathrm{Y}(4260)$ is a $J / \psi f_{J}$ loosely bound molecule (assuming isospin 0). A small admixture thereof on the other hand explains neatly the observed $J / \psi \pi \pi$ decay. This can be excluded by increased statistics for the $\pi \pi$ spectrum. Its proximity to the threshold for $D D_{0,1,2}^{+}$ mesons and other channels shows that a sizable tetraquark or meson molecule should be taken into account in this state's wave function. This seems to be one of the recurrent features of newly found charmed and charmonium states, and its understanding is the most pressing task for theorists.

Also of current interest are states that could contain hidden glue and now we turn to them. The hybrid meson threshold from lattice gauge theory is at around $3.8 \mathrm{GeV}$ [20], from many-body theory at around $4.3 \mathrm{GeV}$ [3]. The found $\mathrm{Y}(4260)$ is close to this second threshold, and the possibility of forming various $L-S$ combinations in a three-body system gives four closely spaced states with various decay patterns (see Fig. 2 in [3]). Still, this assignment presents two problems. To form the quantum numbers $1^{--}$, a $p$ wave is needed either in the $c \bar{c}$ pair (three states) or between this and the hidden gluon (one state). We would then have expected the observed decay channel to be suppressed, against a $\chi_{c} p$-wave meson, as indicated also by the flux tube model This assignment can be ruled out if the (dominant) open charm modes do not contain a $p$-wave meson $D^{+}$. Finally, a fourth low-lying $c \bar{c} g$ state has the $p$ wave between the gluon and the quark-antiquark pair. This state could then have appreciable branching in the observed final-state channel, but the $J / \psi$ would pick up a unit of orbital angular momentum with respect to the pion pair that should also be in a relative $p$ wave to account for parity conservation.

Furthermore, let us observe that in the many-body language, the color octet gluon forces the quark-antiquark pair to be in a color octet too. The repulsive short range interaction then forces them apart and makes the formation of a
$J / \psi$ in the final state unlikely. When decaying to hidden charm states, hybrid mesons would prefer on these general grounds a $p$-wave charmonium. This is consistent with studies of hybrid mesons in the flux tube model and employing lattice adiabatic potentials [21].

The Y(4260) is also massive enough to be a vector glueball. According to lattice studies [22], vector glueballs appear in the spectrum at 3850 (200) MeV. This is in agreement with many-body theory calculations [23], where the minimum Fock space assignment for this odd ball would be a three-gluon state. This option would require a tiny reported leptonic width (hence a large branching fraction in the $J / \psi \pi \pi$ channel). Since the $g g g$ content of the state would be flavor blind, an odd ball would have a large branching ratio to $\phi \pi \pi$. This should be easy to rule out (establish) in the all-charged mode $K^{+} K^{-} \pi^{+} \pi^{-}$with an analysis of the already available data.

\section{CONCLUSIONS}

Pending confirmation of this state, we endorse the $\mathrm{Y}(4260)$ as the $\psi(4260)$, corresponding to the low member of the pair $4 S-3 D$ vector charmonium. To clarify the assignment, we propose that further studies of this state with higher statistics attempt to

(1) discern whether the $\pi \pi$ subsystem is in an $s$ wave relative to the $J / \psi$ instead of a $p$ wave, thus making the $c \bar{c} g$ hybrid assignment unlikely.

(2) Discard the $\phi \pi \pi$ channel typical of flavor-blind odd ball (three-gluon glueball) decay.

(3) Search the $J / \psi \pi^{ \pm} \pi^{0}, J / \psi \pi^{0} \pi^{0}$ channel to determine the isospin. $I \neq 0$ would rule out the simple charmonium assignment and make this state a likely tetraquark candidate.

(4) Increased statistics should allow the identification of the $\psi(4040)$ in the $J / \psi \pi \pi$ spectrum in the same experiment, as it corresponds to the (largely) $3 S$ excitation.

(5) Finally, an attempt to identify $D_{0,1,2}^{+}$mesons is important to identify the role of the nearby $D D^{+}$threshold.

The absence of a $J / \psi K K$ signal at the present statistics is not in conflict with the $4 S$ assignment; the lepton width and mass, although low, are not in blatant disagreement with well-established physics. We finally explain why this resonance has been missed in past searches, being in a readily accessible channel with $1^{--}$quantum numbers, invoking $s$ - and $d$-wave interference in the $D, D_{s}$ form factors.

\section{COMMENT ON OTHER APPROACHES}

Since the first appearance of this report, other authors have exposed their views on this state, mostly suggesting exotic assignments that we now briefly comment on.

In Ref. [24] it has been suggested that a $\chi \rho$ interpretation is likely. We do not see another merit to this conjecture other than the proximity of the relevant threshold, and the 
resonating group method predicts no special attraction in this channel [25] that would suggest a bound state.

The author of Ref. [26] assigns the state to a hybrid multiplet by discarding other possibilities, in particular, the charmonium assignment that we here point out is not unlikely. Further, the authors of [27] support the hybrid assignment. They make use of the fourth hybrid state mentioned above where the $p$ wave is assigned to the gluon. As explained, this assignment is challenged when trying to explain either the $J / \psi \pi \pi$ branching fraction or the coupling to the photon probe, since the $c \bar{c}$ pair is spatially separated. The authors consider the decay of the state into $J / \psi \pi \pi$ by the emission of a second physical gluon. This faces another conceptual difficulty, as the intermediate channel for the decay is a glueball- $J / \psi$ state, at $4.8 \mathrm{GeV}$ by most estimates, which is somewhat off shell and adds to the suppression on the basis of wave function overlap. The selection rule that the authors prove is aimed at suppressing $D \bar{D}$ decay and hence making this state narrow. We just observe that the $s-d$ wave interference mechanism in conventional charmonium achieves a similar effect.

Finally, another article [28] proposes a tetraquark $(c s) \times$ $(\bar{c} \bar{s})$ assignment. Tetraquarks suffer from the well-known problem of state inflation, since there are multiple spin, flavor, color, and spatial wave functions to combine. It is not difficult to find suitable candidates for many nonconventional mesons and a global analysis is necessary to discriminate which states do appear in the spectrum. We do not find compelling the claim about an $f_{0}$ state in the $\pi \pi$ spectrum since this peaks at high available momenta for other processes, it is not visible with the present data, and could point out to a small $J / \psi f_{0}$ admixture and not a tetraquark. More compelling is their prediction $\Gamma_{D_{s} D_{s}} \gg$ $\Gamma_{D D}$, that $B A B A R$ could establish experimentally. For comparison, we plot in Fig. 2 the $D s$ spectrum obtained in a conventional charmonium (with and without interference) and tetraquark models. The latter faces difficulties similar to the other models in terms of explaining a large $J / \psi \pi \pi$

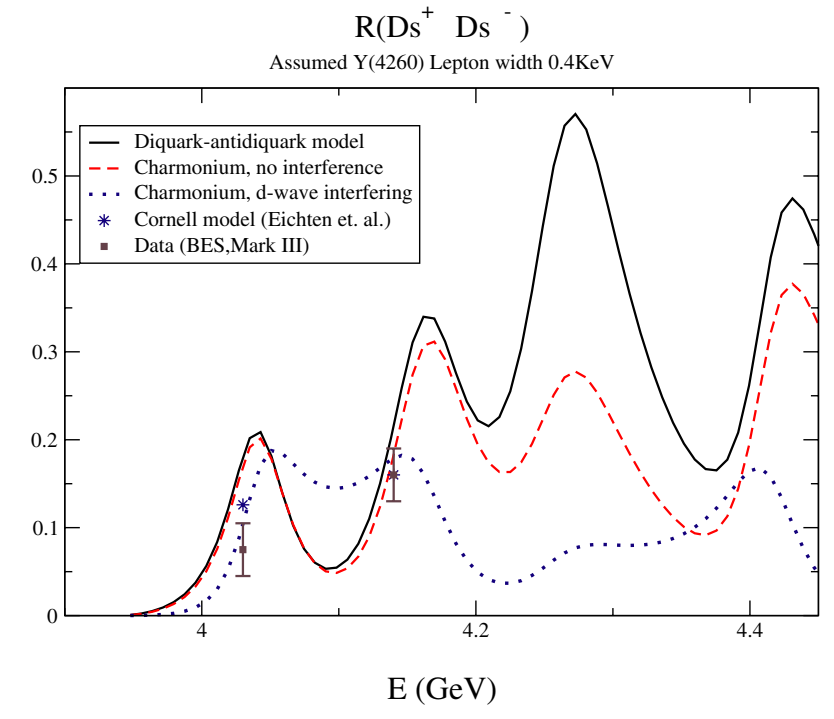

FIG. 2 (color online).

$R^{D_{s} D_{s}}=\left[\sigma\left(e^{-} e^{+} \rightarrow D_{s}^{+} D_{s}^{-}\right) / \sigma\left(e^{-} e^{+} \rightarrow \mu^{+} \mu^{-}\right)\right]$.

width or coupling to a photon probe (since a $p$ wave separates the diquark and the antidiquark).

Therefore we do not see any compelling reason to adopt one of these cryptoexotic model assignments since, on a priori reasons, they do not solve the conceptual problems that this state causes. The last model has the merit of making a prediction that will be tested.

\section{ACKNOWLEDGMENTS}

The author thanks Fundacion del Amo-Universidad Complutense for financial support, the hospitality of the SLAC theory group, and useful conversations with $\mathrm{H}$. Quinn, A. Snyder, K. Yi, U. Mallik, G. Hou, M. Herrero, and W. Dunnwoodie. This work is based on a long and fruitful collaboration with S. R. Cotanch. The work was partly supported by Spanish MCYT Grant No. FPA 200402602 and U.S. DOE Grant No. DE-FG02-97ER41048.
[1] B. Aubert et al. (BABAR Collaboration), hep-ex/0506081 [Phys. Rev. Lett. (to be published)].

[2] S. Godfrey and N. Isgur, Phys. Rev. D 32, 189 (1985).

[3] F. J. Llanes-Estrada and S. R. Cotanch, Phys. Lett. B 504, 15 (2001).

[4] T. Barnes, S. Godfrey, and E. S. Swanson, hep-ph/0505002 [Phys. Rev. D (to be published)].

[5] N. Brambilla et al., hep-ph/0412158.

[6] F. J. Llanes-Estrada and S. R. Cotanch, Nucl. Phys. A697, 303 (2002).

[7] F. J. Llanes-Estrada et al., Phys. Rev. C 70, 035202 (2004).
[8] F. J. Llanes-Estrada and S. R. Cotanch, Phys. Rev. Lett. 84, 1102 (2000).

[9] S. Eidelman et al. (Particle Data Group), Phys. Lett. B 592, 1 (2004).

[10] F. J. Llanes-Estrada and S. R. Cotanch, nucl-th/0408038.

[11] J.Z. Bai et al. (BES Collaboration), Phys. Rev. D 62, 012002 (2000).

[12] E. Eichten et al., Phys. Rev. D 21, 203 (1980).

[13] E. S. Swanson, Phys. Lett. B 598, 197 (2004).

[14] T. Barnes et al., Phys. Lett. B 600, 223 (2004).

[15] A. P. Szczepaniak, Phys. Lett. B 567, 23 (2003). 
[16] P. Bicudo and G. M. Marques, AIP Conf. Proc. 717, 561 (2004).

[17] L. Maiani et al., Phys. Rev. D 70, 054009 (2004).

[18] B. Nicolescu and J.P. B. de Melo, hep-ph/0407088.

[19] U. Dmitrasinovic, Phys. Rev. Lett. 94, 162002 (2005).

[20] X. Liao and T. Manke, hep-lat/0210030.

[21] Katja Waidelich, Masters thesis, North Carolina State University, 2000.

[22] C. J. Morningstar and M. J. Peardon, Phys. Rev. D 60,
034509 (1999).

[23] P. Bicudo et al., SLAC Report No. SLAC-PUB-11312, 2005.

[24] X. Liu, X. Q. Zeng, and X. Q. Li, hep-ph/0507177.

[25] J.E. T. Ribeiro, Z. Phys. C 5, 27 (1980).

[26] S. L. Zhu, hep-ph/0507025.

[27] E. Kou and O. Pene, hep-ph/0507119.

[28] L. Maiani et al., hep-ph/0507062. 